\title{
RELACION ENTRE DEPRESION POST-PARTO Y LA ADHERENCIA A LA LACTANCIA MATERNA EXCLUSIVA EN LAS MADRES QUE ACUDEN AL CONTROL DE CRECIMIENTO Y DESARROLLO EN EL HOSPITAL REGIONAL DOCENTE DE ICA, 2018
}

\author{
RELATIONSHIP BETWEEN POST-PART DEPRESSION AND ADHERENCE TO \\ BREASTFEEDING EXCLUSIVELY IN MOTHERS WHO COME TO CONTROL GROWTH \\ AND DEVELOPMENT IN THE TEACHING REGIONAL HOSPITAL OF ICA, 2018
}

Aucasi-Espinoza Isabel ${ }^{1, a}$, Valencia -Anchante Rody ${ }^{a, b}$

1. Facultad de Medicina. Universidad Nacional San Luis Gonzaga. Ica, Perú.

a. Médico Cirujano.

b. Médico pediatra.

Correspondencia:

Aucasi Espinoza Isabel del Rosario Domicilio: $\quad A v$, pachacutec Yupanqui \#498-Parcona

Teléfono: 944646729

Email:

rosarioaucasi.24@gmail.com

Contribuciones De Autoría:

AEI, VAR: Contribuciones en la concepción y diseño del manuscrito, recolección, análisis e interpretación de los datos redacción y revisión crítica del contenido del manuscrito y la aprobación final del artículo.

Conflicto De Intereses: No declarados.

Financiamiento: Autofinanciado.

\section{Como Citar}

Aucasi-Espinoza Isabel, Valencia -Anchante Rody. Relacion entre depresion post-parto y la adherencia a la lactancia materna exclusiva en las madres que acuden al control de crecimiento y desarrollo en el Hospital Regional docente de Ica, 2018. Rev méd panacea 2019;8(1): 12-20

Recibido: 03 - 03 - 2019

Aceptado: 20 - 04 - 2019

Publicado: 23 - 04 - 2019

\section{RESUMEN}

Objetivo: Establecer la relación entre la depresión post-parto y la adherencia a la lactancia materna exclusiva en las madres que acuden al control de crecimiento y desarrollo en el Hospital Regional Docente de Ica, en el año 2018. Materiales y métodos: Estudio observacional, descriptivo y transversal en 218 madres de lactantes menores de seis meses de edad que acudieron al Servicio de Crecimiento y Desarrollo del Niño del Hospital Regional del Departamento de Ica (Perú). El análisis fue bivariado. Se empleó la prueba chi cuadrado cuando las variables fueron categóricas y la prueba U de Mann Whitney para comparar la mediana de las variables numéricas. Resultados: No existe relación entre la depresión post-parto y la adherencia a la lactancia materna exclusiva. Se encontró relación entre la adherencia a la lactancia materna exclusiva y el orden de nacimiento $\operatorname{del}(a) \operatorname{niño(a)}(p=0,009)$, aseguramiento en salud $(p=0,000)$, actividad laboral $(p=0,041)$, ingreso económico mensual $(p=0,000)$, número de $\operatorname{hijos}(p=0,001)$, tiempo destinado para la crianza y cuidado del niño/a $(p=0,013)$, consejería recibida $(0,000)$ y la autovaloración socio-familiar de las madres $(p=0,001)$. El riesgo de depresión post-parto se asoció a la autovaloración socio-familiar $(p=0,000)$ de las madres que acuden al control de crecimiento y desarrollo en el Hospital Regional Docente de Ica. Conclusiones: No se evidencio relación entre la depresión post-parto y la adherencia a la lactancia materna exclusiva. Sin embargo, el riesgo de depresión post-parto se asoció con la autovaloración socio-familiar.

Palabras clave: Lactancia materna, Depresión postparto.

\section{ABSTRACT}

Objective: To establish the relationship between post-partum depression and adherence to exclusive breastfeeding in mothers attending the control of growth and development in the Regional Teaching Hospital of Ica, in 2018. Materials and methods: Observational, descriptive and transversal study in 218 mothers of infants under six months of age who attended the Child Growth and Development Service of the Regional Hospital of the Department of Ica (Peru). The analysis was bivariate. The chi-square test was used when the variables were categorical and Mann Whitney's U test to compare the median of numerical variables. Results: There is no relationship between postpartum depression and adherence to exclusive breastfeeding. A relationship was found between the adherence to exclusive breastfeeding and the order of birth of the child $(p=0.009)$, health insurance $(p=0.000)$, work activity $(p=0.041)$, monthly income $(p=0.000)$, number of children $(p=0.001)$, time allocated for the upbringing and care of the child $(p=0.013)$, counseling received $(p=0.000)$ and the socio-family self-assessment of the mothers $(p=0.001)$. The risk of post-partum depression was associated with the socio-family self-assessment $(p=0.000)$ of the mothers who come to the control of growth and development in the Regional Teaching Hospital of Ica. Conclusions: There was no evidence of a relationship between postpartum depression and adherence to exclusive breastfeeding. However, the risk of postpartum depression was associated with socio-family self-assessment.

Keywords: Breastfeeding, Depression, Postpartum. 


\section{INTRODUCCIÓN}

La nutrición de los niños durante los primeros meses de vida es crucial porque puede favorecer o limitar su crecimiento físico y desarrollo intelectual en etapas posteriores; además, puede condicionar consecuencias metabólicas importantes durante la infancia tardía y la adolescencia que pueden influir decisivamente en la calidad de vida cuando llegue a la edad adulta (1). Los estudios demuestran que la mayoría de los niños alimentados con lactancia exclusiva durante los primeros seis meses de vida alcanzan un óptimo desarrollo y crecimiento, por lo que no debe ser sustituida con leche artificial y continuar el amamantamiento hasta los dos años de edad (2,3); reduce la mortalidad infantil y ayuda en la prevención de enfermedades de la edad adulta4. En este sentido, la recomendación actual de la OMS para las madres es practicar la lactancia exclusiva desde el nacimiento hasta los seis meses, sin otros líquidos ni alimentos sólidos adicionales que no sean medicamentos o vitaminas $(4,5)$.

A pesar de ser un hecho fisiológico que debería ocurrir con naturalidad, existen factores que pueden condicionar el inicio $\mathrm{y} / \mathrm{o}$ adherencia a esta práctica nutricional. Las madres que en el periodo post-parto temprano tienen dificultades para amamantar tienen mayor tendencia a la depresión post-parto que denota nexos neuroendocrinos que relacionan el aspecto anímico materno post-parto y la lactancia materna $(6,7)$. En este contexto se plantea el presente estudio con el objetivo de determinar la relación entre la depresión post-parto y la adherencia a la lactancia materna exclusiva en las madres que acuden al control de crecimiento y desarrollo en el Hospital Regional Docente de Ica, en el año 2018 (Setiembre-Noviembre).

\section{MATERIALES Y MÉTODOS}

Se realizó un estudio observacional, descriptivo y transversal. La población objetivo estuvo constituida por todas las madres de los y las lactantes menores de seis meses de edad que acuden al Hospital Regional Docente de Ica (Consultorio de Crecimiento y Desarrollo). La muestra fue obtenida mediante muestreo por conveniencia y estuvo conformada por 218 madres de lactantes menores de seis meses de edad. Los criterios de inclusión para esta investigación fueron: Madre que acudió a control de crecimiento y desarrollo de su hijo/a en el Hospital Regional Docente de Ica y aceptación para participar en el estudio mediante consentimiento informado. Los criterios de exclusión fueron Madre con: Demencia, retardo o discapacidad mental, trastorno de personalidad, negativa a continuar en el estudio.

La recolección de datos se realizó a través de una entrevista a la madre de familia del infante. La duración aproximada de esta entrevista fue de 40 minutos. La entrevistadora verificó que todas las preguntas estén respondidas; sin embargo, al finalizar la aplicación de los instrumentos de la investigación (ficha de recolección de datos validad por juicio de expertos y la Escala de
Depresión Post Parto de Edimburgo) realizó el control de calidad previo a la digitación a fin de identificar posibles errores o vacíos. La información resultante de esta investigación fue anónima y manejada confidencialmente por la investigadora. Se solicitó el consentimiento de la madre para participar en el estudio previa información de las características de la investigación y absolución de las preguntas formuladas. Los datos obtenidos, previo control de calidad, fueron ingresados a una base de datos. El procesamiento y análisis se trabajó con un nivel de significancia estadística del 5\%. En el análisis univariado se usó la estadística descriptiva a través de la determinación de proporciones de las variables categóricas, así como las medidas de tendencia central para las variables numéricas. El análisis bivariado se realizó usando la prueba $U$ de Mann Withney para evaluar las diferencias entre las medianas de las variables numéricas; asimismo, se usará la prueba estadística de Chi2 para asociar proporciones con respecto a las variables categóricas, aceptándose como relación estadísticamente significativa si el valor $\mathrm{p}$ fue menor de 0,05

\section{RESULTADOS}

Tabla 1: Características de los niños y madres que acuden al servicio de control de crecimiento y desarrollo. Hospital Regional docente de Ica, 2018.

\begin{tabular}{|c|c|c|}
\hline \multirow{2}{*}{\multicolumn{3}{|c|}{$\begin{array}{l}\text { CARACTERÍSTICAS DEL NIÑO } \\
\text { Sexo }\end{array}$}} \\
\hline & & \\
\hline \multicolumn{3}{|l|}{ Femenino } \\
\hline Masculino & 115 & 50,44 \\
\hline \multicolumn{3}{|c|}{ Orden de nacimiento } \\
\hline Primero & 113 & 49,56 \\
\hline Segundo & 80 & 35,09 \\
\hline Tercero & 17 & 7,46 \\
\hline Cuarto & 8 & 3,51 \\
\hline \multicolumn{3}{|c|}{ Vía de culminación del parto } \\
\hline Abdominal & 44 & 19,3 \\
\hline Vaginal & 174 & 76,32 \\
\hline \multicolumn{3}{|l|}{ CRED a los 7 días } \\
\hline No & 59 & 25,88 \\
\hline Sí & 159 & 69,74 \\
\hline \multicolumn{3}{|c|}{ CARACTERÍSTICAS DE LA MADRE } \\
\hline \multicolumn{3}{|l|}{ Estado civil } \\
\hline Casada & 33 & 14,47 \\
\hline Conviviente & 174 & 76,32 \\
\hline Soltera & 11 & 4,82 \\
\hline \multicolumn{3}{|l|}{ Actividad laboral } \\
\hline Dependiente & 83 & 36,4 \\
\hline Independiente & 26 & 11,4 \\
\hline Ninguna & 109 & 47,81 \\
\hline \multicolumn{3}{|c|}{ Aseguramiento en salud } \\
\hline Privado & 7 & 3,07 \\
\hline EsSalud & 57 & 25 \\
\hline SIS & 78 & 34,21 \\
\hline Ninguna & 76 & 33,33 \\
\hline
\end{tabular}




\begin{tabular}{lcc} 
Tabaquismo & & \\
$\quad$ No & 216 & 94,74 \\
$\mathrm{Si}$ & 2 & 0,88 \\
Inicio de CPN & & \\
$\quad$ No controlada & 7 & 3,07 \\
$\quad$ Tercer trimestre de gestación & 17 & 7,46 \\
$\quad$ Segundo trimestre de gestación & 60 & 26,32 \\
$\quad$ Primer trimestre de gestación & 134 & 58,77 \\
Consejería sobre LME & & \\
$\quad$ No & 131 & 57,46 \\
$\quad$ Si & 87 & 38,16 \\
Valoración socio-familiar & & \\
$\quad$ Riesgo familiar & 55 & 24,12 \\
$\quad$ Problema social & 9 & 3,95 \\
$\quad$ Buena/aceptable situación familiar & 154 & 67,54 \\
\hline
\end{tabular}

En relación a las características de los niños evaluados, 115 (50,44\%) fueron de sexo masculino, 113 (49,56\%) eran el primer hijo y 80 (35,09\%) el segundo hijo. 174 (76,32\%) nacieron por parto vaginal. 159 (69,74\%) tuvieron control de crecimiento y desarrollo a los siete días de edad. En relación a las características maternas, se observó que la mayoría de las madres estaban unidas $174(76,35)$ eran convivientes y $33(14,47 \%)$ eran casadas. 109 (48,80\%) trabajaban en alguna actividad de forma dependiente o independiente, 78 (34,21\%) estaban aseguradas por el SIS y 57 (25,00\%) por EsSalud. Sólo 2 (0,88\%) manifestaron tabaquismo. En 134 (58,77\%) de ellas el control pre-natal se inició en el primer trimestre de la gestación relacionada con el nacimiento del niño índice. 131 (57,46\%) no recibieron consejería sobre lactancia materna mientras que 154 (67,54\%) reportaron buena o aceptable situación familiar (Tabla 1).

Las variables numéricas que caracterizan al niño y a la madre presentaron distribución asimétrica. La mitad de los niños tenían 113 días o menos de nacidos, y 39 o más semanas de edad gestacional al momento de nacer. Asimismo, la mitad de las madres tenía 26 años o más, 11 años de estudios aprobados o menos, ingreso económico igual o superior a 1050 soles, un solo hijo y destinaban 21 horas o menos a la crianza de los niños Tabla 2).

Tabla 2: Distribución de las medidas de tendencia central según características del niño y de la madre que acuden al servicio de control de crecimiento y desarrollo. Hospital Regional docente de Ica, 2018.

\begin{tabular}{|c|c|c|c|c|c|c|}
\hline & $\mathbf{N}$ & Media & $\begin{array}{c}\text { Error } \\
\text { estándar }\end{array}$ & Mediana & Asimetría & Curtosis \\
\hline Edad del niño (días) & 217 & 101760 & 3256 & 113000 & $-0,082$ & $-0,522$ \\
\hline Edad gestacional al nacer (semanas) & 218 & 38427 & 0,105 & 39000 & -2357 & 11763 \\
\hline Edad de la madre (años) & 173 & 26231 & 0,483 & 26000 & 0,430 & $-0,329$ \\
\hline Escolaridad de la madre (años aprobados) & 218 & 11794 & 0,191 & 11000 & $-0,326$ & 1166 \\
\hline Ingreso económico mensual (soles) & 126 & 1329063 & 101758 & 1050000 & 5259 & 40880 \\
\hline Tiempo destinado para la crianza y cuidado del niño/a (horas) & 213 & 18122 & 0,438 & 21000 & $-0,417$ & -1499 \\
\hline Número de hijos & 217 & 1613 & 0,051 & 1000 & 1167 & 1026 \\
\hline
\end{tabular}

Gráfico 1: Depresión post parto en madres que acuden al servicio de control de crecimiento y desarrollo. Hospital Regional docente de Ica, 2018.

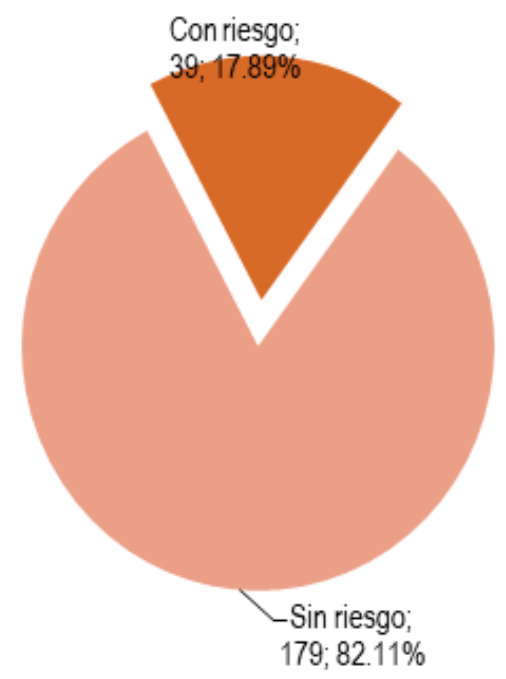

El riesgo de depresión post-parto estuvo presente en 39 (17,89\%) de las madres entrevistadas (Gráfico 1). 
Gráfico 2: Adherencia a la lactancia materna exclusiva en madres que acuden al servicio de control de crecimiento y desarrollo. Hospital Regional docente de Ica, 2018.

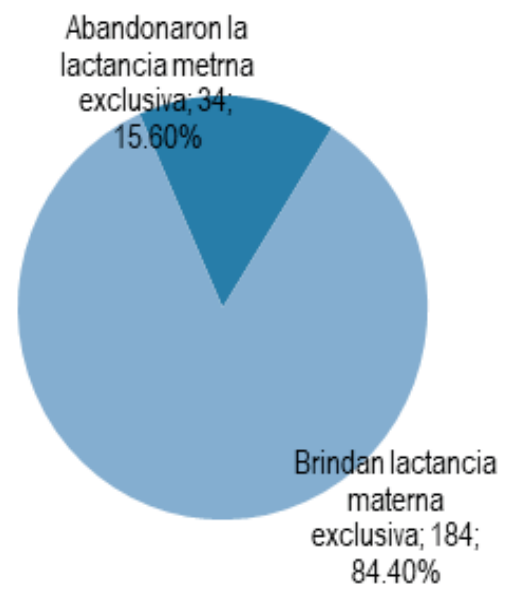

La adherencia a la lactancia materna exclusivo se reportó en 184 (84,40\%) de las madres entrevistadas (Gráfico 2).

De las características del niño, sólo el orden de nacimiento se asoció con la adherencia de la madre a la lactancia materna exclusiva $(p=0,009)$. Hubo mayor porcentaje de abandono de esta práctica por parte de las madres cuando el niño no era el primer nacimiento (Tabla 3).

Tabla 3: Adherencia a la lactancia materna exclusiva en madres según características de los niños y madres que acuden al servicio de control de crecimiento y desarrollo. Hospital Regional docente de Ica, 2018

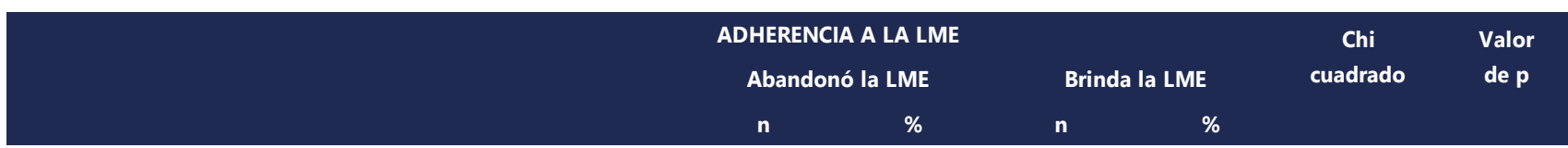

\section{CARACTERÍSTICAS DEL NIÑO}

Sexo

Femenino

18

Masculino

16

Orden de nacimiento

Primero

Segundo

Tercero

Cuarto

Vía de culminación del parto

Abdominal

Vaginal

CRED a los 7 días

No

Sí

CARACTERÍSTICAS DE LA MADRE

Estado civil

Casada

Conviviente

Soltera

Actividad laboral

Dependiente

Independiente

Ninguna
17,48

13,91

9,73

18,75

23,53

50

20,45

14,37

11,86

16,98

19

89
82,52

0,524

0,463
86,21

72,73
0,988

\section{,355}

83,02
0,009

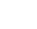

, 32

79,55
85,63

81,65 


\begin{tabular}{|c|c|c|c|c|c|c|}
\hline \multicolumn{7}{|l|}{ Aseguramiento en salud } \\
\hline Privado & 4 & 57,14 & 3 & 42,86 & 32,33 & 0 \\
\hline EsSalud & 1 & 1,75 & 56 & 98,25 & & \\
\hline SIS & 23 & 29,49 & 55 & 70,51 & & \\
\hline Ninguna & 6 & 7,89 & 70 & 92,11 & & \\
\hline \multicolumn{7}{|l|}{ Tabaquismo } \\
\hline $\mathrm{Si}$ & 1 & 50 & 1 & 50 & 0,136 & 0,713 \\
\hline No & 33 & 15,28 & 183 & 84,72 & & \\
\hline \multicolumn{7}{|l|}{ Inicio de CPN } \\
\hline No controlada & 1 & 14,29 & 6 & 85,71 & 5329 & 0,149 \\
\hline Tercer trimestre de gestación & 0 & 0 & 17 & 100 & & \\
\hline Segundo trimestre de gestación & 7 & 11,67 & 53 & 88,33 & & \\
\hline Primer trimestre de gestación & 26 & 19,4 & 108 & 80,6 & & \\
\hline \multicolumn{7}{|l|}{ Consejería sobre LME } \\
\hline No & 9 & 6,87 & 122 & 93,13 & 18,99 & 0 \\
\hline $\mathrm{Si}$ & 25 & 28,74 & 62 & 71,26 & & \\
\hline \multicolumn{7}{|l|}{ Valoración socio-familiar } \\
\hline Problema social & 1 & 11,11 & 8 & 88,89 & 13,11 & 0,001 \\
\hline Riesgo familiar & 17 & 30,91 & 38 & 69,09 & & \\
\hline Buena/aceptable situación familiar & 16 & 10,39 & 138 & 89,61 & & \\
\hline
\end{tabular}

Asimismo, la actividad laboral y el tipo de aseguramiento en salud se asociaron con la adherencia a la lactancia materna exclusiva, aquellas que laboraban de forma independiente o que tenían seguro privado presentaron el mayor porcentaje de abandono ( $26,92 \%$ y $57,14 \%$, respectivamente). Por otro lado, esta adherencia también se relacionó con la consejería recibida sobre LME y con la percepción de la situación socio-familiar $(p<0,05)$ (Tabla 3).

De igual forma, la adherencia a la lactancia materna exclusiva se relacionó con el ingreso económico mensual de la madre, el tiempo destinado para la crianza del niño y con el número de hijos que tenía $(p<0,05)(T a b l a ~ 4)$.

Tabla 4: Comparación de las medidas de tendencia central según características del niño y de la madre que acuden al servicio de control de crecimiento y desarrollo. Hospital Regional docente de Ica, 2018.

\begin{tabular}{|c|c|c|c|c|c|c|}
\hline $\begin{array}{l}\text { Características del niño } \\
\text { y de la madre }\end{array}$ & $\begin{array}{c}\text { Adherencia a } \\
\text { la LME }\end{array}$ & $\mathbf{n}$ & $\begin{array}{c}\text { Rango } \\
\text { promedio }\end{array}$ & $\begin{array}{c}\text { Suma de } \\
\text { rangos }\end{array}$ & $\begin{array}{l}\text { U de Mann- } \\
\text { Whitney }\end{array}$ & Valor de $p$ \\
\hline \multirow[t]{2}{*}{ Edad (días) } & Abandonó & 183 & 106,28 & 19450,00 & 2614000 & 0,139 \\
\hline & Brinda & 34 & 123,62 & 4203,00 & & \\
\hline \multirow[t]{2}{*}{ Edad gestacional al nacer (semanas) } & Abandonó & 184 & 108,74 & 20009,00 & 2989000 & 0,671 \\
\hline & Brinda & 34 & 113,59 & 3862,00 & & \\
\hline \multirow[t]{2}{*}{ Edad de la madre (años } & Abandonó & 139 & 86,41 & 12011,00 & 2281000 & 0,754 \\
\hline & Brinda & 34 & 89,41 & 3040,00 & & \\
\hline \multirow[t]{2}{*}{ Escolaridad de la madre (años aprobados) } & Abandonó & 184 & 111,39 & 20495,50 & 2780500 & 0,246 \\
\hline & Brinda & 34 & 99,28 & 3375,50 & & \\
\hline \multirow[t]{2}{*}{ Ingreso económico mensual (soles) } & Abandonó & 104 & 57,51 & 5981,00 & 521000 & 0,000 \\
\hline & Brinda & 22 & 91,82 & 2020,00 & & \\
\hline \multirow[t]{2}{*}{ Tiempo destinado para la crianza y cuidado del niño/a (horas) } & Abandonó & 182 & 102,95 & 18737,50 & 2084500 & 0,013 \\
\hline & Brinda & 31 & 130,76 & 4053,50 & & \\
\hline \multirow{2}{*}{ Número de hijos } & Abandonó & 184 & 103,61 & 19064,00 & 2044000 & 0,001 \\
\hline & Brinda & 33 & 139,06 & 4589,00 & & \\
\hline
\end{tabular}


Finalmente, se pudo observar que la adherencia a la lactancia materna exclusiva es independiente del riesgo de depresión post-parto en las madres $(p>0,05)$, a pesar que aquellas que tuvieron mayor riesgo de depresión fueron las que presentaron el mayor porcentaje de abandono (Tabla 5).

Tabla 5: Adherencia a la lactancia materna exclusiva según depresión post-parto en madres que acuden al servicio de control de crecimiento y desarrollo. Hospital Rregional docente de Ica, 2018.

\begin{tabular}{|c|c|c|c|c|c|c|}
\hline \multirow{3}{*}{ DEPRESIÓN POST-PARTO } & \multicolumn{4}{|c|}{ ADHERENCIA A LA LME } & \multirow{3}{*}{$\begin{array}{c}\text { Chi } \\
\text { cuadrado }\end{array}$} & \multirow{3}{*}{$\begin{array}{c}\text { Valor de } \\
\quad \mathbf{p}\end{array}$} \\
\hline & \multicolumn{2}{|c|}{$\begin{array}{c}\text { Abandono de la } \\
\text { LME }\end{array}$} & \multicolumn{2}{|c|}{$\begin{array}{l}\text { Brinda } \\
\text { la LME }\end{array}$} & & \\
\hline & $\mathbf{n}$ & $\%$ & $\mathbf{N}$ & $\%$ & & \\
\hline Con riesgo & 9 & 23,08 & 30 & 76,92 & 2019 & 0156 \\
\hline Sin riesgo & 25 & 13,97 & 154 & 86,03 & & \\
\hline
\end{tabular}

Tabla 6: Depresión post-parto en madres según características de los niños que acuden al servicio de control de crecimiento y desarrollo. Hospital Regional docente de Ica, 2018.

\begin{tabular}{cccccccc}
\multicolumn{7}{c}{ DEPRESIÓN POS-PARTO } \\
CARACTERÍSTICAS DE LOS NIÑOS & Con riesgo & Sin riesgo & Chi & Valor de \\
& n & $\%$ & n & $\%$ & cuadrado & p
\end{tabular}

\section{Sexo}

Femenino

Masculino

\section{Orden de nacimiento}

Primero

Segundo

Tercero

Cuarto

\section{Vía de culminación del parto}

Abdominal

Vaginal

\section{CRED a los 7 días}

No

Sí $\begin{array}{llll}16 & 15,53 & 87 & 84,47\end{array}$

$23 \quad 20,00 \quad 92 \quad 80,00$

$0,738 \quad 0,390$

$\begin{array}{llll}21 & 18,58 & 92 & 81,42\end{array}$

$\begin{array}{llll}13 & 16,25 & 67 & 83,75\end{array}$

$\begin{array}{llll}4 & 23,53 & 13 & 76,47\end{array}$

$0,710 \quad 0,871$

112,50

$\begin{array}{ll}7 & 87,50\end{array}$

$\begin{array}{llll}10 & 22,73 & 34 & 77,27\end{array}$

$\begin{array}{llll}29 & 16,67 & 145 & 83,33\end{array}$

$0,878 \quad 0,349$

$\begin{array}{llll}13 & 22,03 & 46 & 77,97\end{array}$

$\begin{array}{llll}26 & 16,35 & 133 & 83,65\end{array}$ 
Ninguna de las características del niño se asociaron con el riesgo de depresión post-parto (Tabla 6), mientras que la valoración socio-familiar fue la única característica materna relacionada de forma significativa con el riesgo de depresión post-parto $(p<0,05)$ (Tabla 7$)$.

Tabla 7: Depresión post-parto en madres según características de las madres que acuden al servicio de control de crecimiento y desarrollo. Hospital Regional docente de Ica, 2018.

\begin{tabular}{cccccccc}
\multicolumn{7}{c}{ DEPRESIÓN POS-PARTO } \\
CARACTERÍSTICAS DE LAS & Con riesgo & Sin riesgo & Chi & Valor de \\
MADRES & $\mathrm{n}$ & $\%$ & $\mathrm{n}$ & $\%$ & cuadrado & $\mathbf{p}$
\end{tabular}

\section{Estado civil}

Casada

Conviviente

Soltera

\section{Actividad laboral}

Dependiente

Independiente

Ninguna

\section{Aseguramiento en salud}

Privado

EsSalud

SIS

Ninguna

\section{Inicio de CPN}

No controlada

Tercer trimestre de gestacić

Segundo trimestre de gesta

Primer trimestre de gestacic

\section{Consejería sobre LME}

No

Valoración socio-familiar

Problema social

Riesgo familiar

$6 \quad 66,67$

$10 \quad 18,18$

$\begin{array}{ll}25 & 19,08 \\ 14 & 16,09\end{array}$

10680,92

$\begin{array}{ll}73 & 83,91\end{array}$

0,319

0,572

Buena/aceptable situación $f$
$3 \quad 33,33$

4581,82

15,500

0,000 
Relacion entre depresion post-parto y la adherencia a la lactancia materna exclusiva en las madres que acuden al control de crecimiento y desarrollo en el Hospital Regional docente de Ica, 2018

\section{DISCUSIÓN}

La lactancia materna, con sus numerosos y conocidos beneficios de salud para los niños y sus madres, es una estrategia clave para mejorar la salud pública. Por ello se recomienda que los bebés sean amamantados exclusivamente durante los primeros 6 meses con leche materna y, luego, continuar junto con la introducción de alimentos complementarios durante al menos dos años $(10,13)$. En nuestro estudio esta práctica fue realizada por cuatro de cada cinco madres entrevistadas, porcentaje superior al reportado por Quispe y col. (14). En un estudio realizado hace seis años en el mismo establecimiento. Este valor es superior a los resultados obtenidos por el Ministerio de Salud en un estudio realizado en cuatro zonas urbanas del Perú (15).

Al igual que en el estudio realizado por Quispe y col.(14), en nuestro estudio, el abandono de la lactancia materna exclusiva no se asoció con el estado civil de la madre, pero sí con el desempeño de alguna actividad laboral por parte de la madre, así como al tipo de la vulnerabilidad socio-familiar y al ingreso económico, aunque estos hallazgos son contradictorios y requieren mayor investigación.

La carga familiar también estaría actuando como elemento que limita la adherencia a la lactancia materna exclusiva debido a que el orden de nacimiento del niño y el mayor número de hijos de la madre aumentó la proporción de abandono de la lactancia materna exclusiva. Pinzón-Villate y col. (15).

También observaron esta asociación en ciudades urbanas de la costa, en aquellas que tenían un solo hijo la adherencia a la lactancia materna exclusiva fue significativamente superior. Posiblemente, mediado por

\section{REFERENCIAS BIBLIOGRÁFICAS}

1. Ahlqvist-Björkroth $S$, Vaarno J, Junttila $N$, Pajulo $M$, Räihä $\mathrm{H}$, Niinikoski $\mathrm{H}$, Lagström $\mathrm{H}$. Initiation and exclusivity of breastfeeding: association with mothers' and fathers' prenatal and postnatal depression and marital distress. Acta Obstetricia et Gynecologica Scandinavica. 2016; 95:396-404. [Citado: Abril 2018 ] Disponible en : https://www.ncbi.nlm.nih.gov/pubmed/26826608

2. Mohamad AS, Tang $L$, Binns CW, Lee $A H$. Breastfeeding and postnatal depression: a prospective cohort study in Sabah, Malaysia. Journal of Human Lactation 2016, 32(2):277-281. [Citado: Marzo 2018] Disponible en: https://sci-hub.tw/10.1177/0890334415620788

3. Pope CJ, Mazmanian D, Bédard M, Sharma V. Breastfeeding and postpDisponibleartum depression: Assessing the influence of breastfeeding intention and other risk factors. Journal of Affective Disorders. 2016; 200:45-50.

32(2):277-281. [Citado: Marzo 2018] Disponible en: https://sci-hub.tw/10.1016/j.jad.2016.04.014

4. Silva CS, Lima MC, Sequeira-de-Andrade LAS, Oliveira JS, Monteiro JS, Lima NMS, etc. Association el mayor tiempo que requiere la crianza de los niños como se puede observar en nuestros hallazgos.

Otro hecho importante es que este abandono estuvo relacionado con la consejería sobre lactancia materna exclusiva después del parto; el porcentaje de abandono fue el triple en aquellas madres que recibieron consejería en comparación con aquellas que no la recibieron; este hecho podría estar traduciendo fallas en esta actividad tan importante para lograr la adherencia a la lactancia materna exclusiva.

Varios estudios han reportado que los síntomas depresivos post-natales se asocian con la ausencia4 o una duración más corta de la lactancia materna exclusiva $(1,2,5,6)$, incluso antes de los dos meses de edad (12), aunque no debe descartarse que la dificultad para la lactancia materna sea un factor de riesgo para depresión post-parto (8) lo que conduciría a las madres a optar por la lactancia materna no exclusiva que es factor asociado a depresión en ellas (9).

Sin embargo, nuestros hallazgos no establecieron asociación entre el riesgo de depresión post-parto y la adherencia a la lactancia materna exclusiva. Pope y col. (3) también han reportado que el intento y la duración de la lactancia no se asocian con la depresión post-parto a los cinco o siete meses después del parto.

Se ha descrito que la depresión post-parto es más probable en madres con menores ingresos, mayor estrés percibido, menor apoyo social percibido, ausencia de antecedentes de depresión o ausencia reciente de antecedentes de abuso (11). Así en nuestro estudio la depresión post-parto se asoció con la valoración de la familia como problema social (4).

between postpartum depression and the practice of exclusive breastfeeding in the first three months of life. J Pediatr (Rio J). 2017; 93(4): 356-364. [Citado: Marzo 2018] Disponible en: http://www.scielo.br/pdf/jped/v93n4/pt_0021-7557jped-93-04-0356.pdf

5. Lara-Cinisomo S, McKenney $\mathrm{K}$, Di Florio $\mathrm{A}$ Meltzer-Brody S. Associations between postpartum depression, breastfeeding, and oxytocin levels in Latina mothers. Breastfeding 2017; 12(7):1-7. [Citado: Mayo 2018] Disponible en: https://sci-hub.tw/10.1089/bfm.2016.0213

6. Zubaran C, Foresti K. The correlation between breastfeeding self-efficacy and maternal postpartum depression in southern Brazil. Sex Reprod Healthc. 2013; 4:9-15. [Citado: Marzo 2018] Disponible en: https://sci-hub.tw/10.1016/j.srhc.2012.12.001

7. Reifsnider $\mathrm{E}$, Flowers J, Todd $\mathrm{M}$, Babendure JB, Moramarco $M$. The relationship between breastfeeding, postpartum depression, and postpartum weight in Mexican American women. J Obstet Gynecol Neonatal Nurs. 2016; 45(6):760-771. [Citado: Mayo 2018] Disponible en: 


\section{REFERENCIAS BIBLIOGRÁFICAS}

https://sci-hub.tw/10.1016/j.jogn.2016.05.009

8. Atencia SM. Factores de riesgo para la depresión posparto en madres adolescentes atendidas en el hospital nacional Daniel Alcides Carrion durante abril - junio, 2015. Lima: Universidad Nacional Mayor de San Marcos. 2015.[Citado: Marzo 2018] Disponible

en: http://cybertesis.unmsm.edu.pe/bitstream/handle/ cybertesis/4355/Atencia_os.pdf?sequence $=1$

9. Yepez HA. Asociación entre lactancia materna no exclusiva y depresión en madres de un hospital público en el Perú. 2017. Lima: Universidad Privada Antenor Orrego. 2018. [Citado: Mayo 2018] Disponible

en: http://repositorio.upao.edu.pe/bitstream/upaorep/ 2608/1/RE_MED.HUMA_HENRY.\%20CASTRO_ASOC IACION.ENTRE.LACTANCIA.MATERNA_DATOS.pdf

10. OMS. Lactancia materna exclusiva. Washington D.C.: Organización Mundial de la Salud. 2017. [Citado: Mayo 2018] Disponible en: http://www.who.int/nutrition/topics/exclusive_brea stfeeding/es/

11. Gorrita RR, Hernández E, Alfonso L. Lactancia materna exclusiva durante el primer semestre de vida, estrés y ansiedad maternos. Medimay. 2017; 24(1):37-52. [Citado: Abril 2018] Disponible en: http://revcmhabana.sld.cu/index.php/rcmh/article/v iew/1042/1448

12. Bao-Alonso MP, Vega-Dienstmaier JM, Saona-Ugarte P. Prevalencia de depresión durante la gestación. Rev Neuropsiquiatr. 2010; 73(3):95-103 [Citado: Mayo 2018] Disponible en: http://www.upch.edu.pe/vrinve/dugic/revistas/index .php/RNP/article/view/1710/1738

13. CDC. Breastfeeding Report Card United States: CDC, 2018. [Citado: Marzo 2018] Disponible en: https://www.cdc.gov/breastfeeding/pdf/2018breastf eedingreportcard.pdf

14. Quispe MP, Oyola AE, Navarro M, Silva JA. Características y creencias maternas asociadas al abandono de la lactancia materna exclusiva. Revista Cubana de Salud Pública. 2015; 41(4):582-592. . [Citado: Abril 2018] Disponible en: http://scielo.sld.cu/pdf/mgi/v33n4/a03_434.pdf

15. Valverde G, Alvis K, Saona R, Pillaca J. Factores asociados a la práctica de lactancia materna exclusiva en madres de zonas urbanas de cuatro provincias del Perú. Lima: Centro Nacional de Alimentación y Nutrición / Instituto Nacional de Salud. 2016. . [Citado: Abril 2018] Disponible en: https://www.mimp.gob.pe/homemimp/direcciones/ diff/ponencias-factores-lactancia.pdf 